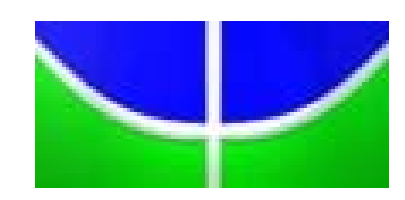

$$
\begin{gathered}
\text { Universidade de Brasília } \\
\text { CET - Centro de Excelência em Turismo }
\end{gathered}
$$

Pós-graduação Lato Sensu

Curso de Especialização em Gastronomia e Segurança Alimentar

\title{
COMPOSIÇÃO NUTRICIONAL DE PREPARAÇÕES EM UM RESTAURANTE DE AUTO-SERVIÇO
}

\section{ELEN MÁRCIA ROCHA DE ABREU}




\section{Universidade de Brasília \\ CET - Centro de Excelência em Turismo}

Curso de Especialização em Gastronomia e Segurança Alimentar

\section{COMPOSIÇÃO NUTRICIONAL DE PREPARAÇÕES EM UM RESTAURANTE DE AUTO-SERVIÇO}

\section{ELEN MÁRCIA ROCHA DE ABREU}

Raquel Botelho, Dra.

Professora Coordenadora
Raquel Botelho, Dra.

Professora Orientadora
Rita Akutsu, Doutoranda

Professora Examinadora

"Trabalho apresentado em cumprimento às exigências acadêmicas parciais do curso de pós-graduação lato sensu em Gastronomia e Segurança Alimentar para a obtenção do grau de Especialista" 
Abreu, Elen Márcia Rocha de

Composição nutricional de preparações em um restaurante de auto-serviço/ Elen Márcia Rocha de Abreu.

Monografia - Curso de Gastronomia e Segurança Alimentar Brasília - DF, abril de 2006.

Área de Concentração: Gastronomia

Orientador: Raquel Botelho

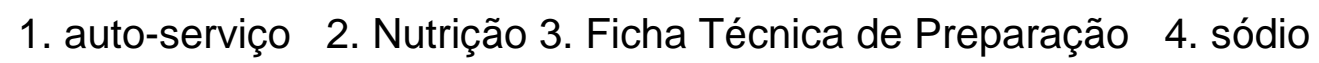

5. Unidades Produtoras de Refeições 6. Composição 
DEDICATÓRIA

Dedico este trabalho à minha mãe, ao meu esposo e aos meus filhos Tiago, Diogo, Renan, Gabriela e Rafael e também à minha irmã Ana Cláudia. Amo vocês! 


\section{AGRADECIMENTOS}

Agradeço à Deus que me conduziu até aqui e por permitir o convívio com pessoas abençoadas que contribuíram para o meu crescimento intelectual e profissional.

À minha orientadora Professora Dra. Raquel Botelho, pela sabedoria, carinho e dedicação durante a realização deste trabalho.

Às minhas amigas Rosana Posse, Ana Carolina Gregório, Lucila Lins e Lígia Cunha que compartilharam as alegrias e dificuldades desta etapa. 


\section{SUMÁRIO}

1. INTRODUÇÃO

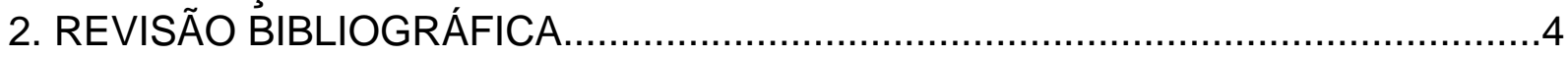

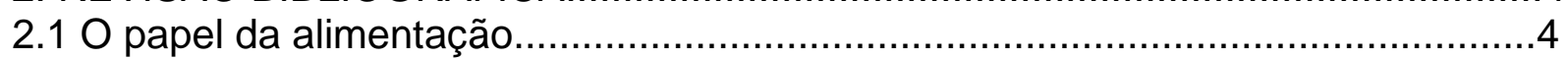

2.2 Hipertensão Arterial e Alimentação...........................................................

2.3 O Monitoramento da alimentação fora do lar como forma de controle da HAS e outras DCNTs

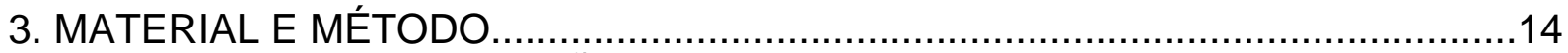

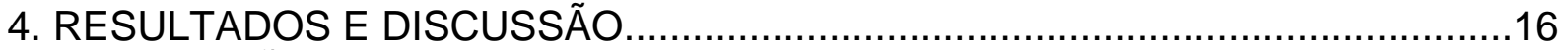

5. CONCLUSÃO REFERÊNCIAS BIBLIOGRÁFICAS APÊNDICES. 


\author{
RESUMO
}

ABREU, Elen Márcia Rocha. Composição nutricional de preparações de um restaurante de auto-serviço, 2006. 90 p. Monografia - Programa de Pósgraduação em Gastronomia, Centro de Excelência em Turismo - UnB. Brasília - DF, 2006.

Orientadora: Dra. Raquel A. Botelho

O modernismo e a globalização trouxeram consigo alterações no estilo de vida da população, que levaram à inclusão das mulheres no mercado de trabalho. Com isto o hábito de realizar as refeições no lar, tornou-se menos freqüente. $O$ mundo moderno e globalizado trouxe mudanças nos hábitos alimentares dos indivíduos, redefinindo o significado da refeição como momento íntimo da reunião dos familiares, por uma alimentação fragmentada, fracionada em pequenas quantidades, em horários determinados pelas atividades individuais dos membros da família, deslocando-se da copa e da cozinha para restaurantes, cafés, automóveis, escritórios, entre outros. Junto com ele, vieram a transição demográfica, nutricional e epidemiológica ocorridas no século passado que determinaram um perfil de risco em que doenças crônicas como a hipertensão assumiram ônus crescente e preocupante. Neste contexto os alimentos oferecidos em unidades produtoras devem ser saudáveis e não oferecer risco à saúde de seus clientes. O presente estudo avaliou quatro preparações, por meio da elaboração de Fichas Técnicas de Preparação, de um restaurante do tipo buffet e auto-serviço do Distrito Federal e identificou-se que todas são hiperlipídicas e possuem um alto teor de sódio, o que poderia prejudicar a saúde das pessoas que se alimentam freqüentemente no local. Além disso, foi verificada a falta de padronização durante o processo de fabricação das mesmas. Sugere-se que as quantidades e os ingredientes sejam revistos e padronizados de forma a promover a saúde e prevenir doenças.

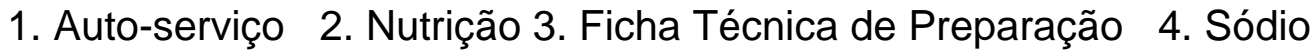
5. Unidades Produtoras de Refeições 6 . Composição 
ABSTRACT

ABREU, Elen Márcia Rocha. Nutritional composition of meals in self-service restaurants, 2006. 90 pages. Monograph - Graduate Program in Gastronomy, Center for Excellence in Tourism - UnB. Brasília - DF, 2006.

Orienting Professor: Dr. Raquel A. Botelho

Modernism and globalization brought about changes in life style. These changes included women in the labor force. So, having meals at home became less frequent. The modern and globalized world caused changes in people's feeding habits, and redefined the meaning of the meal as a close meeting of the family. Meals became fragmented, divided in small portions ingested according the schedules of each family member. Instead of the dining room and the kitchen, food now is consumed in restaurants, cafés, cars, offices, and other places. Together with all that, came the demographic, nutritional, and epidemiological transition that took place in the last century and created a profile of risk in which chronic diseases like high blood pressure took an ever growing and worrying charge. In a context like this, meals offered in producing unities must be healthy, instead of risking their clients' health. This study evaluated four preparations, using Preparation Technical Cards of a self-service restaurant located in the Distrito Federal. The study showed that all of them are hyperlipidemic and present high levels of sodium. Both factors would harm the health of the people that eat frequently in the restaurant. Besides that, the study showed that there is not a standard procedure for the fabrication of meals. The solution appointed is revision and standardizing of quantities and ingredients in order to promote health and avoid diseases.

1. Self-service 2. Nutrition 3. Preparation Technical Cards 4. Sodium 5. Meals Producing Unities 6. Composition 


\section{INTRODUÇÃO}

O modernismo e a globalização trouxeram consigo alterações no estilo de vida da população, que levaram à inclusão das mulheres no mercado de trabalho. Com isto o hábito de realizar as refeições no lar, tornou-se menos freqüente (VEIROS; PROENÇA, 2003).

Comer fora do lar não é apenas uma necessidade de quem vive e trabalha nas grandes cidades. É, na verdade, uma opção para as famílias, seja pela facilidade ou pela qualidade da alimentação oferecida. Com as opções de lugares para comer fora de casa e de serviços de entrega em domicílio, sem mencionar a propagação dos alimentos pré-cozidos e congelados, esta prática torna-se um novo comportamento do brasileiro interferindo no ramo da alimentação que se apresentam como um mercado em expansão (PROENÇA, 2002).

Deve-se ressaltar que o crescimento do consumo de alimentos fora do lar tornou-se uma prática muito constante na população, pela praticidade, variedade e falta de tempo de deslocamento no horário de refeições (JOMORI et al., 2005).

Os dados da Associação Brasileira de Bares e Restaurantes-ABRASEL, em 2004, mostraram que diariamente, cerca de 45 milhões de brasileiros se alimentam fora de casa. O segmento de "food service" cresceu $15,5 \%$ contra $9,7 \%$ do varejo. De acordo com a associação, daqui a 15 anos, o percentual de gastos com a alimentação fora de casa corresponderá a $40 \%$ de toda a despesa com comida.

Ocorre que essa realidade vem de encontro às expectativas interrelacionadas ao setor de turismo que é o segmento responsável por $40 \%$ do PIB e por $53 \%$ da mão-de-obra empregada, o que está diretamente ligado ao desenvolvimento do país, por meio do crescimento planejado e estruturado da área de alimentação fora do lar (ABRASEL, 2006).

Segundo a ABRASEL (2004), até meados dos anos 80, eram raras as opções

para quem precisava almoçar fora de casa. As alternativas eram restritas a restaurantes com serviço "à la carte", lanchonetes ou pequenos estabelecimentos comerciais que ofereciam o prato feito, conhecido popularmente por "PF". 
Neste sentido a instalação de redes de "fast-food" e o surgimento dos restaurantes "self-service" por quilo (auto-serviço) possibilitaram maior variedade na escolha entre pratos de saladas, carnes e massas, pagando apenas pelo consumo.

O conceito de restaurante do tipo "self-service" está ligado a uma oferta de preparações muito além daquelas que habitualmente são consumidas nos lares. Vários fatores interferem na escolha destes alimentos no momento da refeição. Dentre eles o fator cultural, considerando a variedade, quantidade e qualidade de ingredientes utilizados nos restaurantes. Neste sentido pode-se afirmar que os alimentos essenciais à vida humana possuem características que podem ser prejudiciais à saúde, tanto pela falta quanto pelo excesso do consumo desses alimentos (VEIROS; PROENÇA, 2003).

Contudo, a alimentação é uma necessidade biológica, consciente e voluntária que envolve aspectos relacionados à sua condição de onívoro, ou seja, comer de tudo, sendo condicionada por aspectos antropológicos, biológicos, sócio-culturais e econômicos que são fundamentais no momento da escolha alimentar (SENNA; ARAÚJO, 2004).

Alimento, segundo Senna e Araújo (2004), é todo material que o ser humano recebe com a finalidade de obtenção de nutrientes para suprimento de energia corporal. Assim sendo, torna-se essencial o investimento em estudos de análise quantitativa e qualitativa dos ingredientes, por meio de verificação das fichas técnicas de preparações (FTPs) em estabelecimentos que oferecem refeições fora do lar.

Com esta preocupação, é que se torna relevante o estudo da composição das preparações e conseqüentemente das FTPs e da sua fidedignidade em estabelecimentos comerciais.

Este estudo tem como objetivo geral avaliar a quantidade de sódio em preparações oferecidas em um restaurante com sistema de "buffet" e auto-serviço. Os objetivos específicos englobam a verificação da existência de FTPs no estabelecimento; elaboração e avaliação das referidas FTPs das preparações selecionadas; verificação dos temperos utilizados; e investigação do percentual de sódio com auxílio do detector de sódio.

Para melhor estruturar a compreensão deste trabalho, o mesmo foi dividido em introdução que contempla influências da modernização e da globalização nos 
hábitos alimentares, principalmente em relação ao consumo de alimentos fora do lar; revisão bibliográfica, destacando o papel da alimentação no contexto da manutenção da vida; a alimentação e suas implicações no controle da hipertensão arterial; materiais e métodos utilizados neste trabalho; discussão e resultados obtidos e conclusão. 


\section{REVISÃO BIBLIOGRÁFICA}

\subsection{O PAPEL DA ALIMENTAÇÃO}

Os alimentos são substâncias líquidas ou sólidas que, levadas ao trato digestivo, são metabolizadas e produzem energia para a manutenção da vida (SESI, 2003). Desta forma a alimentação constitui uma das atividades mais importantes do ser humano, que é influenciada por fatores biológicos e sócio-culturais da população (PROENÇA, 2005).

A longevidade e qualidade de vida vêm de encontro a uma alimentação equilibrada, contendo todos os nutrientes necessários à nutrição do organismo. Portanto, uma dieta em qualidade e quantidade suficientes se faz necessária para manter um funcionamento perfeito do corpo e um bom estado de saúde (MONDINI; MONTEIRO, 1994).

Os hábitos alimentares são respostas às regras culturais, que exercem grande influência sobre o comportamento da população que segundo Sloan (2005) é o que determina como fazer, como servir e como comer os alimentos.

Michaelis (2005) define cultura como "sistema de idéias, conhecimentos, técnicas e artefatos, de padrões de comportamento e atitude que caracteriza uma determinada sociedade". Agregando-se a esta definição, Araújo et al (2005) definem cultura como complexidade de padrões de comportamento, crenças, instituições, valores espirituais e materiais transmitidos coletivamente e característicos de uma sociedade.

Partindo do princípio de que a cultura é um padrão de comportamento, podese afirmar que cultura é também um hábito, que pode ser definido como uso ou costume, disposição para alguma coisa, adquirida pela freqüente repetição dos mesmos atos, podendo ou não fazer parte da cultura. O mesmo conceito pode ser estendido para comportamento alimentar, que são influenciados por vários fatores que determinam as decisões alimentares (GARCIA, 2003).

O comportamento alimentar sofre grande influência da família, que é responsável pela aquisição e preparo dos alimentos, conforme a sua cultura e as transformações do mundo globalizado.

A diversidade de transformações inclui o alto consumo de refeições fora do lar. Atualmente nunca se consumiu tanto fora de casa. Este fato se equipara ao 
crescimento da oferta de vários restaurantes, com estilos variados; desde os "fast foods" até os sofisticados, que visam principalmente à satisfação das necessidades e desejos dos clientes (SLOAN, 2005), sem priorizar a alimentação saudável e equilibrada, condições primordiais para a saúde.

Este crescimento deve-se aos modernos padrões de vida, onde a população possui pouco tempo para desenvolver muitas atividades e não se reserva um momento durante o dia para se alimentar de forma saudável e tranqüila. Há grande procura pelos restaurantes do tipo "self-service", pois os alimentos já estão preparados e expostos, bastando serem escolhidos e servidos para serem consumidos.

A tendência da evolução de padrões dietéticos tem sido avaliada com base em dados sobre disponibilidade de alimentos, que são compilados anualmente pela Organização das Nações Unidas para a Alimentação e Agricultura (FAO). Os dados indicam a quantidade potencial média de alimentos disponível para consumo humano em cada país, considerando produção, importação e exportação de produtos alimentares e um percentual arbitrário de desperdício, que talvez seja a única fonte regular e padronizada de informações sobre padrões alimentares (MONDINI; MONTEIRO, 1994).

Os mesmos autores destacam as Pesquisas de Orçamento Familiar (POFs) como fonte alternativa de informações sobre consumo alimentar, que é estimado por meio de despesas efetuadas com os alimentos e preços praticados no comércio, que são essenciais para a identificação de mudanças em padrões de consumo alimentar no Brasil.

O mundo moderno e globalizado trouxe mudanças nos hábitos alimentares dos indivíduos, redefinindo o significado da refeição como momento íntimo da reunião dos familiares, por uma alimentação fragmentada, fracionada em pequenas quantidades, em horários determinados pelas atividades individuais dos membros da família, deslocando-se da copa e da cozinha para restaurantes, cafés, automóveis, escritórios, entre outros (ORTIZ, 1994).

Ortiz (1994) relata que neste contexto de transformações, verifica-se que um dos principais requisitos na escolha dos restaurantes é a rapidez no servir e no 
comer como forma de adaptação a este novo estilo de vida que permeia a população.

Fazendo uma análise antropológica superficial sobre a alimentação, verificase que é originária do universo doméstico e está envolta no convívio familiar e social, diretamente ligada a figura feminina, seja da mulher ou da mãe, que são referências afetivas. Neste contexto, a alimentação era compartilhada essencialmente no lar. Mesmo com a inserção da mulher no mercado de trabalho, além de provedora, continua sendo de sua responsabilidade a administração do lar, que vai desde a adequação do cardápio aos recursos financeiros, à realização das compras e o preparo das refeições (GARCIA, 1997).

A valorização de uma profissionalização feminina representada pelo trabalhar fora colocou a necessidade da diminuição do tempo de preparo do alimento ou mesmo de sua eliminação através da procura por alimentos feitos fora de casa, congelados ou não, acompanhando um movimento mundial (DUTRA, 1991).

Em contrapartida, não se pode atribuir o aumento do consumo de alimentos fora do lar somente pelo fato da mulher estar trabalhando fora de casa durante maior tempo. Há outros fatores considerados relevantes: a distância entre o local de trabalho e a casa, as dificuldades de deslocamento pelo trânsito e ainda o intervalo de tempo destinado para a alimentação também interferem nesta escolha (JAMORI, 2005).

Segundo estimativas da Associação Brasileira das Indústrias da Alimentação (ABIA), aponta-se, nos centros urbanos, que 30\% das refeições são realizadas fora do lar e o crescimento do setor é promissor. Pesquisas apontam que em um shopping center de porte médio em São Paulo, circulam cerca de 40.000 pessoas ao dia, sendo que 10 a $20 \%$ freqüentam as praças de alimentação, ou seja, em torno de 4.000 a 8.000 pessoas consomem alimentação fora do lar diariamente (YAMAMOTO et al., 2004).

Este novo estilo de vida traz os maus hábitos alimentares que influenciam no funcionamento do organismo como um todo, provocando conseqüências maléficas que podem ser, muitas vezes, irreversíveis; tanto pelo excesso quanto pela falta de nutrientes. Reconhecendo essa influência negativa da dieta no estado de saúde dos indivíduos, a Organização Mundial da Saúde (OMS) estabeleceu guias alimentares, 
que definem limites seguros para o consumo dos nutrientes (MONDINI; MONTEIRO, 1994).

Estes guias alimentares trazem parâmetros quantitativos seguros para o consumo de macronutrientes, micronutrientes, vitaminas e minerais. Os excessos no consumo desses nutrientes vêm contribuindo para o desenvolvimento de doenças crônicas não-transmissíveis (DCNTs).

As transições demográfica, nutricional e epidemiológica ocorridas no século passado determinaram um perfil de risco em que doenças crônicas como a hipertensão assumiram ônus crescente e preocupante (TOSCANO, 2004).

A importância das DCNTs no perfil atual de saúde das populações é extremamente relevante. Estimativas da OMS apontam que as DCNT já são responsáveis por $58,5 \%$ de todas as mortes e por $45,9 \%$ da carga total global de doenças expressa por anos perdidos de vida saudável (MONTEIRO et al., 2005).

Dentre elas a hipertensão arterial é um problema crônico comum. Sua prevalência é alta e aumenta em faixas etárias maiores. Estudos epidemiológicos brasileiros estimam prevalências de $40 \%$ a $50 \%$ de hipertensão na população adulta com mais de 40 anos, a partir da medida casual da pressão (FUCHS, 1994). Mesmo sendo assintomática, a hipertensão arterial é responsável por complicações cardiovasculares, renais, entre outras. Em virtude da hipertensão ser assintomática, parcela importante da população adulta com hipertensão não sabe que é hipertensa; e muitos dos que sabem não estão sendo adequadamente tratados (TOSCANO, 2004).

Mondini e Monteiro (1994) ressaltam que há uma estreita relação entre características qualitativas da dieta e a ocorrência das DCNTs, assim como das doenças cardiovasculares e diferentes tipos de câncer. Os autores apontam a relação da composição lipídica do alimento com a obesidade. Há evidências de que a obesidade possa se relacionar à proporção de energia proveniente de gorduras, independentemente do total calórico da dieta. 


\subsection{HIPERTENSÃO ARTERIAL E ALIMENTAÇÃO}

Conforme o relatório mundial de saúde de 2002, a maior parte dos fatores de riscos para DCNT inclui a hipertensão arterial sistêmica-HAS, que é desencadeada por vários fatores e dentre eles destaca-se o alto consumo de alimentos ricos em sal e a obesidade.

Dados encontrados em estudos cuja finalidade era conhecer a epidemiologia da doença hipertensiva demonstraram grande variação de percentual de indivíduos portadores de HAS. É desconhecido o número exato de indivíduos hipertensos no país, mas de acordo com os dados disponíveis, a prevalência de hipertensão arterial no Brasil varia de $14 \%$ a $47,9 \%$ da população (BRANDÃo et al., 2003).

Em estudos populacionais realizados em alguns estados brasileiros, no período de 1990 a 2001 e divulgados pelo Departamento de Hipertensão Arterial da Sociedade Brasileira de Cardiologia, demonstraram a prevalência da HAS conforme figura 1.

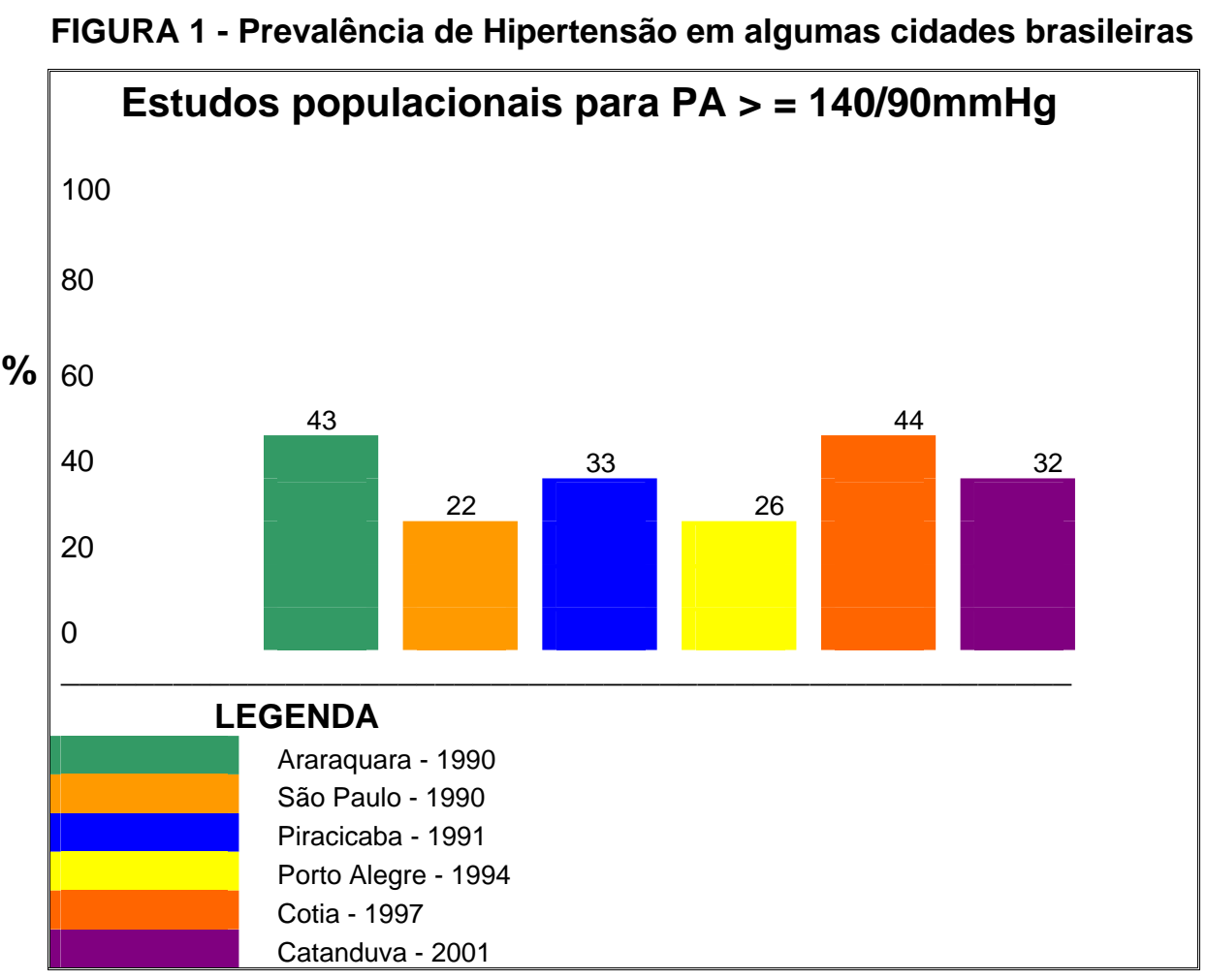

Fonte: IV Diretrizes Brasileiras de Hipertensão

A HAS é modernamente entendida como uma doença inserida em um contexto mais abrangente do que apenas aquele resultante da simples definição dos 
níveis pressóricos, hoje considerados por muitos como um critério intermediário dentro de um quadro mais amplo. Cifras pressóricas elevadas são reconhecidas como marcadores de risco cardiovascular, porém isoladamente carecem de suficiente sensibilidade e especificidade (BRANDÃO et al., 2003).

Os mesmos autores relatam que o conceito atualmente difundido é de uma condição sistêmica que envolve a presença de alterações estruturais das artérias e do miocárdio, associadas à disfunção endotelial e à constrição e remodelamento da musculatura lisa vascular. Condições estas que estão amplamente relacionadas à presença de fatores de risco como distúrbios metabólicos ligados à obesidade, ao diabete e às dislipidemias, entre outros.

As diretrizes definem a HAS em adultos, quando a pressão sistólica se encontra em níveis iguais ou acima de $140 \mathrm{mmHg}$ e/ou quando a pressão arterial diastólica for igual ou maior que $90 \mathrm{mmHh}$ em duas ou mais ocasiões, na ausência de uso de medicação anti-hipertensiva. O parâmetro de normalidade compreende em valores inferiores a 130/85 mmHg e ótima quando for igual ou inferior a 120/80 mmHg (BRANDÃO et al., 2003). (figura 2).

FIGURA 2: Classificação das HAS pela IV Diretrizes Brasileiras de Hipertensão

\begin{tabular}{||lcc||}
\hline \multicolumn{3}{|c||}{ Classificação da PA ( $>18$ anos) } \\
Classificação & PAS (mmHg) & PAD(mmHg) \\
Ótima & $<120$ & $<80$ \\
Normal & $<130$ & $<85$ \\
Limítrofe & $130-139$ & $85-90$ \\
& & \\
Hipertensão & $140-159$ & $90-99$ \\
Estágio I (leve) & $160-179$ & $100-109$ \\
Estágio II (moderado) & $>=180$ & $<90$ \\
Estágio III (grave) & $>=140$ & $<10$ \\
Sistólica isolada & & \\
& & \\
O valor mais alto de sistólica ou diastólica estabelece o estágio do quadro \\
hipetensivo. Quando as pressões sistólica e diastólica situam-se em categorias \\
diferentes, a maior deve ser utilizada para classificação do estágio.
\end{tabular}

Fonte: IV Diretrizes Brasileiras de Hipertensão

Estes dados remetem à necessidade de reavaliação dos componentes das receitas culinárias, como forma de quantificar e qualificar os ingredientes utilizados, 
uma vez que a prevenção e o tratamento da HAS estão diretamente relacionados com a quantidade de sódio diário ingerido.

Foi identificado em sites de gastronomia e livros de técnicas culinárias que na maioria das receitas, há ingredientes como realçadores de sabores, caldos concentrados de diversos tipos e outros condimentos que em sua composição química contém grande percentual de gordura e de sal, que são agregados aos temperos utilizados no dia-a-dia, proporcionando mais sabor às preparações.

Ocorre que, os restaurantes buscam oferecer uma alimentação com sabores rebuscados como forma de atração e fidelização de sua clientela, sem a preocupação com o valor calórico e, conseqüentemente, nutricional da refeição. $O$ excesso de ingredientes como o sal e a gordura podem desencadear o sobrepeso e a obesidade, além do aumento da pressão arterial (TOSCANO, 2004).

O sal é definido como termo químico para substância produzida pela reação de um ácido com uma base. Quando o sódio, um metal instável que pode inflamarse subitamente, reage com o cloro, gás cuja toxicidade pode ser letal, o resultado é o cloreto de sódio $(\mathrm{NaCl})$, alimento básico proveniente da única família de rocha que os seres humanos comem, cujo sabor é o que se chama de salgado (KURLANSKI, 2004).

Segundo Kurlanski (2004), o cloreto de sódio é essencial para o funcionamento do organismo humano. Possui papel importante na digestão e respiração, no transporte de nutrientes e oxigênio, na transmissão de impulsos nervosos e movimentação dos músculos, incluindo o coração.

O mesmo autor relata que um adulto contém em média, $250 \mathrm{~g}$ de sal. Esta quantidade é utilizada pelas funções corporais que ocasionam sua redução constante, e por isto precisa ser reposta.

Kurlanski (2004) enfatiza que o sal é uma substância forte e às vezes perigosa. Tal afirmativa justifica a preocupação dos cientistas quanto a quantidade segura de ingestão do sal.

Em função disso, o relatório do Institute of Medicine (IOM) estabeleceu o AI (Adequate Intake), ou seja, estimativa da ingestão diária para o sal em 3,8 g/dia (1,5 $\mathrm{g}$ de sódio e 2,3 $\mathrm{g}$ de cloreto) para balancear as perdas de sódio no suor em adultos. Porém pessoas fisicamente ativas têm uma necessidade aumentada de sal: "Esta Al 
não se aplica a indivíduos muito ativos que perdem grandes quantias de suor diariamente" (IOM, 2004, p. 61).

É preciso lembrar que a deficiência deste nutriente causa cefaléia e fraqueza, depois tontura e náusea; persistindo a privação por muito tempo pode provocar morte.

\footnotetext{
"Mas em nenhum momento desse processo há o desejo de comer sal. No entanto, a maioria das pessoas opta por comer muito mais sal do que precisa, e pode ser que essa vontade - o simples fato de gostar do sabor do sal - seja uma defesa natural" (KURLANSKI, 2004, p. 27).
}

Estes dados confirmam a necessidade em adoção de medidas preventivas quanto a ingestão diária de sal, uma vez que as alterações iniciais da pressão arterial são freqüentemente associadas a presença de outros fatores de risco cardiovasculares.

As doenças cardiovasculares representam nacional e internacionalmente um importante problema de saúde pública, que a pelo menos três décadas são a primeira causa de morte no Brasil conforme o Sistema de Informação sobre Mortalidade - SIM (BRANDÃO et al., 2003).

No Brasil, as doenças cardiovasculares corresponderam a mais de $27 \%$ do total de óbitos, responsáveis por 15,2\% das internações realizadas no Sistema Único de Saúde - SUS, em 2000. Do total de casos quantificado em 693.839, 17,7\% foram relacionados ao acidente vascular encefálico e ao infarto do miocárdio, doenças de grande importância epidemiológica e que têm a HAS como um de seus principais fatores de risco (BRANDÃo et al., 2003).

Não há dados brasileiros consolidados em relação ao montante de recursos envolvidos no tratamento, controle e suporte previdenciário da doença. Segundo o Sistema de Informação Hospitalar (SIH/SUS), foram gastos R $\$ 17$ milhões em internações por HAS no ano de 2000 (BRANDÃO et al., 2003).

Com tantos estudos relevantes e dada à importância negativa dos efeitos da ingestão inadequada de sódio na saúde humana, torna-se essencial o controle da utilização do sal no preparo das refeições produzidas em restaurantes comerciais, visando à oferta de alimentos mais saudáveis. 
2.3 O MONITORAMENTO DA ALIMENTAÇÃO FORA DO LAR COMO FORMA DE CONTROLE DA HAS E OUTRAS DCNTS.

É extremamente rica a literatura sobre a ingestão de Eletrólitos na dieta, sobretudo o sódio do sal de cozinha. Os dados existentes envolvem considerações antropológicas que indicam que a adição de sal ao alimento é recente na história da humanidade, relacionada à necessidade de conservação do mesmo, e não relacionada às necessidades dietéticas do eletrólito (LOLIO, 1990).

Neste sentido as fichas técnicas de preparação (FTPs) constituem um instrumento útil e essencial para auxiliar na montagem de cardápios nutricionalmente mais adequados às necessidades da clientela. Além disso, permite maior controle de custos e padronização das preparações.

A arte culinária é milenar. Desde os ancestrais já se faziam experiências com as diversas combinações de alimentos que eram disponíveis na natureza, até mesmo antes da descoberta do fogo, que marca o surgimento da culinária. A aceitabilidade das preparações era avaliada por meio dos efeitos agradáveis ou desagradáveis que os mesmos proporcionavam após sua ingestão (TEICHMANN, 2000). Não foram encontrados relatos sobre a existência de FTPs, nesta época. De forma que as refeições não tinham um padrão.

O padrão, segundo Akutsu et al (2005) "é um compromisso documentado, utilizado em comum e repetido muitas vezes pelas pessoas relacionadas com uma determinada função." Ou seja, deve ser elaborado com a participação de quem executa o trabalho, e, a partir deste ponto, deve ser seguido por todos.

A padronização dos serviços visa à capacitação eficiente e eficaz dos envolvidos na função, além de proporcionar maior controle de custos. As vantagens são inúmeras e dentre elas pode-se citar que para o cliente a garantia de obtenção do produto com as mesmas características sensoriais, que envolvem o sabor, a textura, o aroma e cor. Para a empresa uma é ferramenta facilitadora para o treinamento dos funcionários, reduz o retrabalho, melhora a supervisão realizada pelo gerente ou nutricionista, entre outros (AKUTSU et al., 2005).

Os mesmos autores mencionam que parte dos problemas em organizações brasileiras deve-se aos funcionários que trabalham em diferentes turnos, quando 
executam uma mesma tarefa de formas diferentes, ocasionando variações no processo produtivo e conseqüentemente na qualidade.

A qualidade no seguimento alimentação envolve desde o ambiente até a atração pelo produto exposto, que deve ser seguro do ponto de vista higiênicosanitário e satisfazer as expectativas dos clientes de forma constante. A utilização de FTPs é uma das ferramentas que pode ser utilizada para a garantia da qualidade (AKUTSU et al., 2005).

Akutsu et al (2005) ressaltam que as FTPs são facilitadoras do processo produtivo, que trazem informações sobre: per capita, fator de correção e cocção, composição centesimal em micro e macronutrientes da preparação, tempo gasto com o pré-preparo e preparo das refeições, e o rendimento e porcionamentos das mesmas. Ou seja, é um instrumento controlador da produção.

Concomitante $a$ isso, os dados sobre os ingredientes utilizados em cada preparação, quantidades e técnica de preparo constantes nas FTPs possibilitam o controle financeiro e dos gêneros, e o cálculo do cardápio (AKUTSU et al., 2005).

A partir das FTPs é possível a realização do planejamento do cardápio com maior controle. Vale ressaltar que, desde que elaboradas adequadamente, suas informações e instruções permitirão a racionalização do processo de produção.

Desta forma, o planejamento do cardápio deve contemplar o uso das FTPs, que possibilita obter informações sobre: per capita, fator de correção e cocção, composição centesimal em macro e micronutrientes da preparação, tempo para o pré-preparo e preparo, rendimento e porcionamento das refeições. A partir destes dados é possível a padronização e controle para a monitorização durante o processo produtivo, quanto a escolha e/ou oferta de opção alimentares mais adequadas. 


\section{MATERIAL E MÉTODO}

O presente trabalho é um estudo do tipo exploratório e descritivo. Foi selecionada uma Unidade Produtora de Refeições com sistema de buffet e autoserviço no Distrito Federal, configurando-se uma amostra de conveniência.

De acordo com Diehi e Tatim (2004) as pesquisas exploratórias são indicadas para proporcionar maior familiaridade com o problema, para torná-lo mais explícito ou a construir hipóteses, que envolve levantamento bibliográfico, além de realizar a pesquisa juntamente com pessoas que possuem experiência prática com o problema pesquisado. Ao final da análise, proporcionar maiores informações sobre $o$ assunto e identificar formas de intervenção nas questões em estudo. A associação entre uma pesquisa exploratória e descritiva confirma-se pela utilização de técnicas padronizadas durante a coleta de dados e a observação sistemática durante o processo.

O restaurante possui o sistema de distribuição centralizado do tipo buffet e a modalidade de atendimento é o auto-serviço. Possui um cardápio misto prevalecendo as preparações formais e atende uma clientela seleta de maior poder aquisitivo.

Para a realização da pesquisa no estabelecimento, foi feito um contato prévio com o proprietário, que liberou o acesso às dependências do restaurante. Após o consentimento, foram realizadas as visitas para a coleta de dados e posterior análise.

Para cada preparação foram coletados os dados durante três dias diferentes e, após elaborar as FTPs realizou-se a média aritmética para análise das quantidades.

Foram escolhidas, aleatoriamente, quatro preparações para análise, sendo dois pratos principais (Filé à parmegiana e Rosbife ao molho madeira), uma guarnição (Purê de batata) e um acompanhamento (Arroz à piamontese).

Durante o trabalho foi necessário realizar as FTPs de ingredientes-base (arroz branco, roux, fundo de carne, tempero da casa, molho de tomate e molho madeira) para produção das preparações previamente escolhidas, para viabilizar o estudo. 
As FTPs (anexo 1) foram desenvolvidas durante o acompanhamento minucioso dos processos de pré-preparo e preparo, para mensuração dos ingredientes. Foram quantificados com auxílio de balanças digitais: uma da marca Plenna modelo TIN-00910 peso máximo 10kg, precisão de $1 \mathrm{~g}$ para garantir a fidedignidade dos dados. Durante a verificação dos processos, foi observado se existia um ou mais cozinheiros em cada estabelecimento e também se o mesmo profissional utiliza algum padrão para mensuração dos ingredientes.

Além das etapas acompanhadas, foram coletados e calculados os dados de fator de cocção, rendimento e modo de preparo.

O fator de cocção foi calculado dividindo-se o peso da preparação cozida pelo somatório dos ingredientes crus.

Após a conclusão da preparação, utilizou-se um detector de sódio eletrônico marca Lumiscope ${ }^{\circledR}$, Modelo 3011, para verificar o percentual de sódio contido nas preparações. Os dados foram analisados, avaliados e comparados com os dados da tabela de composição de alimentos para demonstração dos resultados observados.

Após estes procedimentos e com a gramatura de todos os ingredientes, os valores nutricionais das preparações foram calculados com base em Tabelas de Composição Química dos Alimentos (Franco, ano; Pinheiro et al, ano; IBGE, ano) utilizando-se para este fim o programa de planilhas eletrônicas Microsoft Excel.

As porções médias foram estimadas a partir do relato da funcionária que realiza a reposição das preparações do "buffet".

O cálculo de cada FTP contemplou o valor energético total (VET), os macronutrientes: carboidratos (CHO), proteínas (PTN) e lipídios (LIP) e o micronutriente sódio (Na). Após o cálculo de cada uma das triplicatas das FTPs de cada preparação, a média dos valores nutricionais foi calculada, avaliando as diferenças percentuais. 


\section{RESULTADOS E DISCUSSÃO}

No total, foram acompanhadas quatro preparações, sendo que para cada uma, os dados foram coletados durante três dias não consecutivos. No entanto, os dados de um dia de análise tanto do Rosbife ao molho madeira quanto do Filé à parmegiana foram descartados visto que houve erros durante a coleta que inviabilizaria um resultado confiável.

Dessa forma, para a média aritmética e posterior análise da composição nutricional das preparações, considerou-se duas FTPs do Rosbife ao molho madeira e do Filé à parmegiana e três para o Purê de batata e Arroz à piamontese.

$\mathrm{Na}$ Tabela 1, pode-se observar a diferença percentual entre os dois dias de análise do Rosbife ao molho madeira, em relação à composição nutricional.

\begin{tabular}{|l|c|c|c|}
\hline \multicolumn{4}{|c|}{$\begin{array}{l}\text { Tabela 1. Diferença percentual da composição nutricional entre os dois } \\
\text { dias de análise do Rosbife ao molho madeira }\end{array}$} \\
& $\mathbf{1}^{\mathbf{0}}$ dia de análise & $\mathbf{2}^{\mathbf{0}}$ dia de análise & Percentual \\
\hline VET TOTAL (Kcal): & 660,57 & 559,55 & $15,29 \%$ \\
\hline PTN (g): & 34,67 & 30,24 & $12,76 \%$ \\
\hline LIP (g): & 55,23 & 46,97 & $14,95 \%$ \\
\hline CHO (g): & 6,21 & 3,95 & $36,31 \%$ \\
\hline Na (mg): & 579,29 & 590,17 & $1,88 \%$ \\
\hline
\end{tabular}

Para a avaliação, foi considerada a mesma porção (110g), sendo possível observar que o valor calórico e os macronutrientes do primeiro dia de análise foram superiores ao segundo, no entanto, quanto ao sódio, foi inferior $(1,88 \%)$.

Provavelmente esta pequena diferença de sódio se deve ao fato de a carne ser a principal fonte de sódio da preparação.

Comprova-se por meio dos valores encontrados que a despadronização das quantidades e modo de preparo influencia não somente no custo e nas características sensoriais do produto, como também na composição nutricional.

Em relação à análise do Filé à parmegiana, observa-se na Tabela 2 que houve uma diferença percentual mais expressiva na quantidade de carboidrato $(37,41 \%)$ e de sódio $(28,37 \%)$, para uma mesma porção de 150g.

A quantidade de tempero, de queijo mussarela e de ingredientes para empanar (ovo, farinha de trigo e farinha de rosca) foi maior que o utilizado no primeiro dia, considerando que ambos produziram praticamente a mesma 
quantidade de porções $(8,25$ x 8,19). Isso justificaria o maior valor encontrado de carboidrato e sódio. Outra probabilidade da diferença de sódio é a adição de realçador de sabor (Ajinomoto) no preparo do molho de tomate, parte integrante na montagem do prato, do segundo dia, que não foi utilizado no preparo do mesmo molho, no primeiro dia.

Porém, quanto ao valor calórico e os macronutriente proteína e lipídio, a diferença percentual foi menor, se comparada à preparação anterior.

\begin{tabular}{|c|c|c|c|}
\hline & $1^{\circ}$ dia de análise & $2^{\circ}$ dia de análise & Percentual \\
\hline VET TOTAL (Kcal): & 361,79 & 379,82 & $4,98 \%$ \\
\hline PTN (g): & 19,87 & 20,72 & $4,27 \%$ \\
\hline LIP (g): & 26,33 & 26,07 & $0,98 \%$ \\
\hline $\mathrm{CHO}(\mathrm{g}):$ & 11,34 & 15,58 & $37,41 \%$ \\
\hline $\mathrm{Na}(\mathrm{mg})$ : & 292,78 & 375,83 & $28,37 \%$ \\
\hline
\end{tabular}

As diferenças percentuais do Purê de batata, para uma mesma porção de $90 \mathrm{~g}$, entre os três dias de análise foi realizada comparando-se o $1^{\circ}$ e $2^{\circ}$ dia, o $1^{\circ}$ e $3^{\circ}$ dia e o $2^{\circ}$ e $3^{\circ}$ dia, de forma que fosse possível identificar em qual dos dias os valores percentuais foram mais discrepantes.

\begin{tabular}{|c|c|c|c|c|c|c|}
\hline & $\begin{array}{l}1^{\circ} \text { dia de } \\
\text { análise }\end{array}$ & $\begin{array}{c}2^{\circ} \text { dia de } \\
\text { análise }\end{array}$ & $\begin{array}{c}3^{\circ} \text { dia de } \\
\text { análise }\end{array}$ & $\begin{array}{c}\text { Percentual } \\
\text { entre } 1^{\circ} \text { e } 2^{\circ} \\
\text { dia de análise }\end{array}$ & $\begin{array}{c}\text { Percentual } \\
\text { entre } 1^{\circ} \text { e } 3^{\circ} \text { dia } \\
\text { de análise }\end{array}$ & $\begin{array}{c}\text { Percentual } \\
\text { entre } 2^{\circ} \text { e } 3^{\circ} \\
\text { dia de análise }\end{array}$ \\
\hline VET TOTAL (Kcal): & 80,00 & 80,00 & 79,99 & $0,00 \%$ & $0,01 \%$ & $0,01 \%$ \\
\hline PTN (g): & 3,05 & 3,07 & 2,96 & $0,81 \%$ & $2,76 \%$ & $3,54 \%$ \\
\hline LIP (g): & 3,88 & 4,08 & 4,52 & $4,98 \%$ & $16,34 \%$ & $10,83 \%$ \\
\hline CHO (g): & 8,21 & 7,75 & 6,87 & $5,60 \%$ & $16,39 \%$ & $11,43 \%$ \\
\hline $\mathrm{Na}(\mathrm{mg}):$ & 49,67 & 49,47 & 46,83 & $0,40 \%$ & $5,72 \%$ & $5,34 \%$ \\
\hline
\end{tabular}

Observa-se na primeira análise, que o lipídio e o carboidrato foram os que apresentaram maior diferença percentual (4,98\% e 5,6\%, respectivamente). No primeiro dia, foi utilizada a mesma quantidades de manteiga e uma maior de creme de leite, se comparado ao segundo, para produzir diferentes porções $(56,42 \times$ $52,44)$, o que justifica o percentual encontrado. 
Quanto ao carboidrato, pode ser justificado pela diferença encontrada entre os "per capitas" líquidos de batata entre o primeiro e segundo dia ( $46 \mathrm{~g} \times 44 \mathrm{~g})$. No entanto, apesar das diferenças encontradas na gramatura dos macronutrientes, 0 valor calórico permaneceu o mesmo para as estes dois dias analisados

Em relação ao primeiro e terceiro dia, as diferenças percentuais foram mais expressivas que a da análise anterior, sendo que no lipídio, no carboidrato e no sódio foram, respectivamente, 16,34\%, 16,39\% e 5,72\%.

Quanto ao lipídio, pode ser justificado pelo uso de uma quantidade superior de manteiga no terceiro dia, em relação ao primeiro, sendo que neste último, 0 número de porções produzidas foi superior ao outro $(56,42 \times 47,47)$.

Os valores diferentes de per capita líquido da batata do primeiro com 0 terceiro dia $(46 \mathrm{~g} \times 39 \mathrm{~g})$ foram os responsáveis por esta diferença percentual em relação ao carboidrato.

O "per capita" de sal foi maior no primeiro dia de análise, em relação ao terceiro, sendo este o fator da diferença percentual encontrada, visto que para esta preparação foi utilizada manteiga sem sal e que o per capita do queijo parmesão foi praticamente o mesmo para os dois dias.

Ainda em relação ao sódio, porém comparando-se o segundo e o terceiro dia, observou-se que, pelo mesmo motivo do citado acima a diferença percentual demonstrou que há maior quantidade de sódio por porção no segundo dia, se comparado ao outro.

No segundo dia, o per capita líquido da batata foi de $44 \mathrm{~g}$ e, no terceiro, $39 \mathrm{~g}$, 0 que provocou a diferença percentual do carboidrato (11,43\%). Em relação ao lipídio, assim como explicado na análise entre o primeiro e terceiro dia, o per capita da manteiga foi maior no terceiro, em comparação ao segundo dia $(1,23 \mathrm{~g} \times 1,76 \mathrm{~g})$.

Observando-se a Tabela 3, verifica-se que as principais diferenças percentuais foram referentes ao carboidrato e ao lipídio, que em resumo, atribui-se a despadronização da quantidade de batata e manteiga, respectivamente.

A avaliação da porção $(90 \mathrm{~g})$ dos três dias de análise do Arroz à piamontese está descrita na Tabela 4. 
Tabela 4. Diferença percentual da composição nutricional entre os três dias de análise do Arroz à piamontese

\begin{tabular}{|c|c|c|c|c|c|c|}
\hline & $\begin{array}{c}1^{\circ} \text { dia de } \\
\text { análise }\end{array}$ & $\begin{array}{c}2^{\circ} \text { dia de } \\
\text { análise }\end{array}$ & $\begin{array}{l}3^{\circ} \text { dia de } \\
\text { análise }\end{array}$ & $\begin{array}{c}\text { Percentual } \\
\text { entre } 1^{\circ} \text { e } 2^{\circ} \\
\text { dia de análise }\end{array}$ & $\begin{array}{c}\text { Percentual } \\
\text { entre } 1^{\circ} \text { e } 3^{\circ} \\
\text { dia de análise }\end{array}$ & $\begin{array}{c}\text { Percentual } \\
\text { entre } 2^{\circ} \text { e } 3^{\circ} \\
\text { dia de análise }\end{array}$ \\
\hline VET TOTAL (Kcal): & 195,24 & 189,28 & 192,04 & $3,05 \%$ & $1,64 \%$ & $1,46 \%$ \\
\hline PTN (g): & 4,05 & 3,98 & 4,21 & $1,80 \%$ & $3,82 \%$ & $5,73 \%$ \\
\hline LIP (g): & 8,99 & 9,08 & 9,40 & $-0,99 \%$ & $4,59 \%$ & $3,57 \%$ \\
\hline \begin{tabular}{|l|}
$\mathrm{CHO}(\mathrm{g}):$ \\
\end{tabular} & 24,53 & 22,92 & 22,65 & $6,58 \%$ & $7,68 \%$ & $1,17 \%$ \\
\hline $\mathrm{Na}$ (mg): & 415,29 & 443,98 & 425,98 & $-6,91 \%$ & $2,57 \%$ & $4,06 \%$ \\
\hline
\end{tabular}

Quanto à diferença percentual de sódio (6,91\%) entre o primeiro e o segundo dia, verificou-se que o per capita do realçador de sabor (Ajinomoto) foi o provável responsável pelo resultado e, em relação ao carboidrato (6,58\%), no primeiro dia, a porção de arroz cozido foi maior que no segundo $(73,91 \mathrm{~g} \times 68,82 \mathrm{~g})$.

Analisando-se as diferenças percentuais dos outros dias, concluir-se que a porção do arroz branco, o per capita do creme de leite e o do realçador de sabor foram determinantes para o resultado, considerando, respectivamente, os valores de carboidrato, lipídio e proteína e, por fim, o sódio.

Para analisar o Valor Energético Total (VET), percentual de macronutrientes e quantidade de sódio por porção, de cada preparação, foi realizada a média aritmética dos valores. Os resultados estão descritos na tabela abaixo (Tabela 5).

Tabela 5. Composição nutricional por porção das preparações analisadas

\begin{tabular}{|l|c|c|c|c|c|c|}
\hline \multicolumn{1}{|c|}{ Preparação } & $\begin{array}{c}\text { Porção } \\
\text { (g) }\end{array}$ & $\begin{array}{c}\text { VET } \\
\text { (Kcal) }\end{array}$ & $\begin{array}{c}\text { Proteína } \\
\text { (\%) }\end{array}$ & $\begin{array}{c}\text { Lipídio } \\
\text { (\%) }\end{array}$ & $\begin{array}{c}\text { Carboidrato } \\
\text { (\%) }\end{array}$ & $\begin{array}{c}\text { Na } \\
\text { (mg) }\end{array}$ \\
\hline Filé à parmegiana & 150 & 371,0 & 21,38 & 62,08 & 14,17 & 334,44 \\
\hline Purê de batata & 90 & 80,0 & 15,14 & 46,81 & 38,05 & 48,66 \\
\hline Rosbife ao molho madeira & 110 & 610,1 & 21,25 & 75,37 & 3,38 & 584,73 \\
\hline Arroz à piamontese & 90 & 192,19 & 8,50 & 42,87 & 48,63 & 428,42 \\
\hline
\end{tabular}

O Filé à parmegiana é uma preparação que deveria ser fonte de proteína, porém de acordo com o resultado, o percentual protéico representa apenas 21,38\% e o lipídico, 62,08\%, caracterizando-a como hiperlipídica. Segundo a OMS (2003) o percentual máximo recomendado de lipídeos é de 30\% nas refeições. Desta forma, o cliente terá dificuldade de equilibrar seu prato quando optar por se servir de Filé à parmegiana. 
Em relação ao Rosbife ao molho madeira, este deveria apresentar características de uma preparação protéica, o que não ocorreu. O percentual de lipídio encontrado foi de $75,37 \%$ e o valor calórico também foi um resultado expressivo, já que se encontrou $610 \mathrm{Kcal}$ numa porção de $110 \mathrm{~g}$ desta preparação. Pode-se atribuir os resultados encontrados ao molho madeira, uma vez que em sua composição adicionou-se manteiga, fundo de carne (molho base) e o roux (composto por $50 \%$ de margarina e $50 \%$ de farinha de trigo). Foi a preparação mais calórica e lipídica encontrada, não devendo ser servida todos os dias no estabelecimento. Outra opção seria modificar a receita para que fosse diminuída a quantidade de gordura.

O purê de batata, por ser uma guarnição, deveria apresentar principalmente, um percentual de carboidratos dentro da faixa preconizada que é de $50 \%$ a $60 \%$ (OMS, 2003), sendo que a base da preparação é a batata inglesa. No entanto o resultado demonstra que o percentual lipídico $(46,81 \%)$ é maior que o de carboidratos (38,05\%) e o protéico está acima do esperado, podendo ser justificado pela presença de ingredientes como o queijo parmesão e o creme de leite, além da manteiga, que possuem alto teor de lipídios e de proteínas.

Assim como as demais preparações, o Arroz à piamontese apresentou características hiperlipídicas (42,87\%) e não exerceu a função de um acompanhamento, , que deveria ter maior percentual de carboidrato (48,63\%). Em sua preparação, foram utilizados creme de leite, gema de ovo, manteiga e queijo parmesão, que contribuíram para o resultado encontrado. Em receitas pesquisadas na Internet, na lista de ingredientes do arroz a piamontesa não há gema de ovo, o que poderia ser retirado da preparação sem nenhum prejuízo de sabor; o que tornaria o prato menos calórico e com um percentual lipídico menor.

Considerando que o almoço deve suprir 40\% das necessidades diárias (FAUSTO, 2003) e que o consumo de sódio diário não deve ultrapassar 2400 mg (American Heart Association, 2000), determinou-se que, para a avaliação do teor de sódio, fosse considerado um valor máximo de 960mg para esta refeição.

Desta forma, a análise foi baseada no percentual que cada preparação representa dentro do limite de sódio previamente determinado (Figura 3). 
Figura 3. Adequação das preparações em relação ao valor máximo de $\mathrm{Na}$ consumido no almoço.

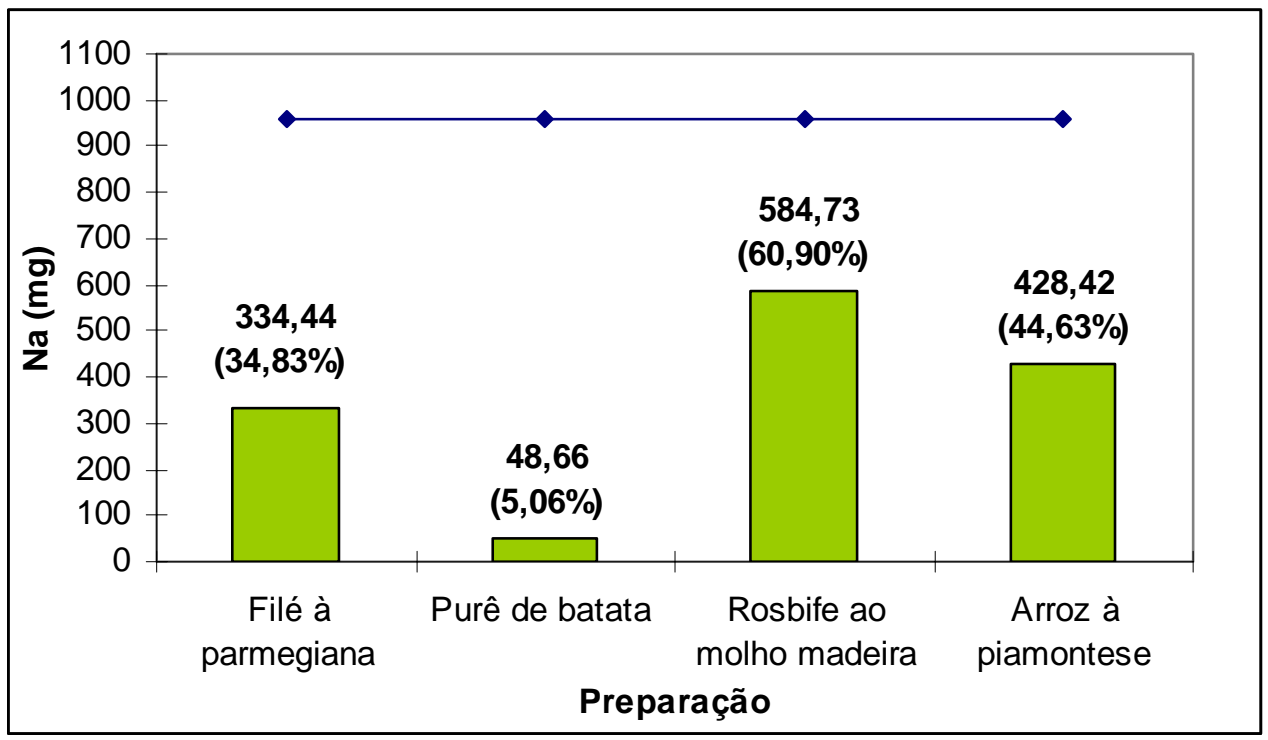

Observa-se que o Rosbife ao molho madeira, o Arroz à piamontese e o Filé à parmegiana possuem um teor de sódio muito alto, se for considerado que, caso o cliente consuma uma porção de um dos pratos principais mais o Arroz à piamontese, o percentual de sódio ultrapassa o limite determinado ou mesmo fica muito próximo dele (representado na figura pela linha reta acima das barras $=960 \mathrm{mg}$ de sódio). No entanto, sabe-se que em um restaurante de auto-serviço, a variedade de alimentos é muito grande e a possibilidade de um cliente se servir de outras preparações é alta.

Baseado nos cálculos das fichas técnicas e considerando que o restaurante é de auto-serviço, pode-se afirmar que os clientes desta unidade de produção estão consumindo sódio em excesso, o que pode causar hipertensão arterial ou mesmo agravar a doença nos que a possuem, conforme relatado nos estudos descritos na revisão bibliográfica.

O uso de realçador de sabor (Ajinomoto), temperos prontos (fondor), fundo de carne (molho base), o próprio sal e o tempero da casa foram utilizados nas preparações. No entanto, pode-se sugerir que se acrescente ervas aromáticas e outros temperos como pimenta-do-reino, de forma que realce o sabor das preparações sem que seja necessário acrescentar essas diversas fontes de sódio.

Tanto 0 sal quanto o realçador de sabor (Ajinomoto) contribuem significativamente para o aumento do teor de sódio nas diferentes preparações. 
Porém o realçador de sabor tem uma parcela maior. Confere-se nas embalagens dos realçadores de sabores que em $100 \mathrm{~g}$ do produto há $123 \mathrm{mg}$ de sódio. Identificou-se na pesquisa que este produto é muito utilizado, e que o cozinheiro desconhece as propriedades do produto, fazendo uso indiscriminado em várias preparações.

A relação entre o teor de sódio encontrado pelo detector de sódio e o encontrado por meio de cálculos utilizando as tabelas de composição química dos alimentos está representado na Tabela 6 abaixo. Os dados demonstraram que os valores encontrados utilizando-se o detector de sódio foram superiores aos encontrados por meio dos cálculos.

\begin{tabular}{|c|c|c|}
\hline $\begin{array}{l}\text { Tabela 6: Representação } \\
\text { tabelas e o detector de sć }\end{array}$ & ça entre o sódio enc & do por meio \\
\hline PREPARAÇÃO & $\begin{array}{c}\text { DETECTOR DE SÓDIO } \\
(\mathrm{g})\end{array}$ & $\begin{array}{c}\left(^{*}\right) \text { TABELA } \\
(\mathrm{g})\end{array}$ \\
\hline Filé à parmegiana & 1,1 & 0,33 \\
\hline $\begin{array}{l}\text { Rosbife de filé ao molho } \\
\text { madeira }\end{array}$ & 1,1 & 0,58 \\
\hline Purê de batatas & 0,83 & 0,05 \\
\hline Arroz a piamontese & 0,5 a 1,0 & 0,43 \\
\hline
\end{tabular}

(*) Cálculos realizados por meio de tabelas de composição química dos alimentos.

Outro dado observado é a diferença entre os ingredientes das receitas consideradas padrão, das praticadas no restaurante. Observou-se que no arroz a piamontese há adição de gema de ovo na preparação, o que não acontece na receita padrão; no purê de batatas acrescenta-se o queijo parmesão, que também não consta nos ingredientes da receita padrão. Além disto, o molho base e o tempero caseiro é preparado no estabelecimento, nos quais é utilizada grande quantidade de realçador de sabor (ajinomoto).

Por não haver no estabelecimento fichas técnicas de preparação, torna-se inviável o controle dos custos de produção, a padronização nos sabores de cada preparação e consequentemente na qualidade da alimentação oferecida pelo restaurante. Sendo um restaurante de auto-serviço com balcão livre, os clientes podem se servir à vontade o que pode colocar em risco a sua própria saúde, conforme as escolhas dos diversos pratos com a mesma característica: hiperlipídicos e hipercalóricos. 
Por todos os motivos apresentados, sugere-se a elaboração e implantação das fichas técnicas de preparação e a busca por receitas tradicionais, onde não há adição de ingredientes que tornam a preparação nutricionalmente inadequada. 


\section{CONCLUSÃO}

Este estudo foi importante para confirmar que ainda há uma lacuna a ser preenchida unindo a gastronomia e a segurança alimentar e nutricional, pois considerando ambos essenciais, devem proporcionar melhor qualidade na alimentação, tanto no aspecto sensorial como na promoção da saúde e prevenção de doenças.

Diante da transição demográfica e o alto índice de doenças crônico-nãotransmissíveis, como a hipertensão arterial, confirma-se a necessidade em adoção de medidas preventivas quanto a ingestão diária de sal, uma vez que as alterações iniciais da pressão arterial são freqüentemente associadas a presença de outros fatores de risco cardiovasculares e ainda pelo fato de ser assintomática.

Com tantos estudos relevantes e dada à importância negativa dos efeitos da ingestão inadequada de sódio na saúde humana, torna-se essencial o controle da utilização do sal no preparo das refeições produzidas em restaurantes comerciais, visando à oferta de alimentos mais saudáveis.

Neste estudo, das quatro preparações avaliadas, somente o Purê de batata não apresentou valor alto de sódio, porém nenhuma delas seria recomendável para uma dieta considerada saudável, já que todas se apresentaram hiperlipídicas.

Sugere-se que as preparações sejam revistas de forma a modificar tanto qualitativo quanto quantitativamente os ingredientes de forma que sejam um fator de risco à saúde dos clientes do restaurante em questão.

Além destes aspectos, também verificou-se que a falta de padronização nos processos produtivos não é um fator positivo para o estabelecimento, visto que interfere diretamente nos custos das preparações e, conseqüentemente no lucro, apesar deste não ter sido um fator avaliado. As características sensoriais e a composição nutricional também se alteram.

Logo, a Ficha Técnica de Preparação surge como um instrumento de padronização e avaliação da receita e sugere-se que, antes de ser implementada sejam feitas as alterações sugeridas neste trabalho. 


\section{REFERÊNCIAS BIBLIOGRÁFICAS}

AMERICAN HEART ASSOCIATION. AHA Scientific Statement. Dietary guidelines. Revision 2000. A statement for health care professionals from the nutrition committee of the American Heart Assocition. Circulation 2000, n¹02, p. 2284-99, 2000.

ARAÚJO, C.M.W; BOTELHO, R.;GINANI, C.V; ARAÚJO, C.M.H; ZANDONADI, P.R. Da Alimentação a gastronomia. 1a . Edição. Ed. Universidade de Brasília. Brasília, 2005.

BRANDÃO, A. P.; BRANDÃO, A. A.; MAGALHÃES, M. E. C.; POZZAN, R. Epidemiologia da Hipertensão Arterial. Rev. Soc. Cardiologia Estado de São Paulo. Vol. 13 - nr. 1, Jan/Fev., 2003.

DUTRA, M. R. C. A Boa Mesa Mineira; um estudo de cozinha e identidade, Dissertação de mestrado apresentada ao Programa de Pós-Graduação do Museu Nacional da UFRJ, 1991.

GUIMARÃES, A. et al. Matéria de capa. Tendência do Food Service: Oferecer Alimentação Saudável. Rev. Nutrição em Pauta, São Paulo, Ano IX, n 74, Mar/Abr, p. 8-14, 2001.

. Uma questão para empresários e consumidores. 2006. Disponível em www.abrasel.com.br. Acesso em 05/04/2006. (Paulo Solmucci Júnior)

$\overline{04 / 02 / 2006 .}$

Entrevista .... 2004. Disponível em www.abrasel.com.br. Acesso em

FAUSTO, M.A. Planejamento de dietas e da alimentação. Rio de Janeiro - RJ: Editora Revinter, 2003.

FUCHS FD, MOREIRA LB, MORAES RS, BREDEMEIER M; CARDOZO SC. Prevalence of systemic arterial hypertension and associated risk factors in the Porto Alegre metropolitan area. Populational-based study. Arquivos Brasileiros de Cardiologia 63(6):473-479.

GARCIA, R. W. D. Práticas e comportamento alimentar no meio urbano: um estudo no centro da cidade de São Paulo. Cad. Saúde Pública, Rio de Janeiro, 13 (3), p. 455-467, jul-set, 1997. 
GARCIA, R. W. D. Reflexos da Globalização na Cultura Alimentar: considerações sobre as mudanças na alimentação urbana. Rev. de Nutrição, Campinas, 16 (4), p. 483-494, out-dez, 2003.

http://departamentos.cardiol.br/dha/ivdiretriz/slides/slides.asp

Institute of Medicine (2004). Dietary Reference Intakes for water, potassium, sodium, chloride, and sulfate. Washington, DC: The National Academies Press, in press. (www.iom.edu/report.asp?id=18495). Rev. Gatorade Sports Science Institute. vol 41 Set-Out-Nov 2004. Requerimentos Nutricionais de água e sódio para adultos ativos..

JOMORI, M. M., et al. Proposta de um Método para a avaliação da Escolha Alimentar do Comensal de um Restaurante por Peso. Rev. Nutrição em Pauta, São Paulo, Ano XIII, nº 74, Set/Out, p. 45-48, 2005.

LOLIO, C. A. de. EPIDEMIOLOGÍA DA HIPERTENSÃO ARTERIAL. Rev. Saúde Pública. São Paulo 24 (5): 425-32, 1990.

MONDINI L.; MONTEIRO, C. A. Mudanças no padrão de alimentação da população urbana brasileira (1962-1988). Rev. Saúde Pública, 28 ("): 433-9, 1994. São Paulo.

MONTEIRO, C. A; MOURA, E. C; JAIME, A. L; FLORINDO, A. A; FIGUEIREDO, I. C. R; BERNAL, R; SILVA, N. N. Monitoramento de Fatores de Risco para doenças crônicas por entrevistas telefônicas. Rev. de Saúde Pública 2005; 39(1): 47-57.

ORGANIZAÇÃO MUNDIAL DE SAÚDE. Diet, nutrition and prevention of chronic diseases. Report of a joint WHO/FAO expert consultation. Technical Report Series 916. Geneva, 2003.

ORTIZ, R. Mundialização e Cultura, São Paulo: Brasiliense, 1994.

PROENÇA, R. P. da C. Desafios Contemporâneos com Relação à Alimentação Humana. Rev. Nutrição em Pauta, São Paulo, Ano X, nº. 52, Jan/Fev, p. 32-36, 2002.

PROENÇA, R. P. da C. Qualidade Nutricional e Sensorial na Produção de Refeições. Rev. Nutrição em Pauta, São Paulo, Ano XIII, n. 75, Nov/Dez, p. 4-14, 2005.

SENNA, T.; Araújo, W. M. C. Avaliação do hábito de lavar as mãos entre consumidores de restaurantes do tipo self service. Trabalho apresentado no I Congresso Brasileiro de Gastronomia. CET - UnB, 2004). 
SESI - Departamento Regional de São Paulo. Alimente-se bem com R\$1,00. 6 . Edição. Julho 2003. São Paulo.

SLOAN, D. [tradução Sonia Bidutte] Gastronomia, restaurantes e comportamento do consumidor. Barueri, SP: Manole, 2005.

TEICHMANN, I.M. Cardápios - técnicas e criatividade. 5. ed. Caxias do Sul: EDUCS, 2000. Coleção Hotelaria.

TOSCANO, C.M. As campanhas nacionais para detecção das doenças crônicas não-transmissíveis: diabetes e hipertensão arterial. Rev. Ciência e Saúde Coletiva, 9(4): 885-895, 2004.

VEIROS, M. da B., PROENÇA, R. P. da C. Avaliação Qualitativa das Preparações do Cardápio em uma Unidade de Alimentação e Nutrição - Método AQPC. Rev. Nutrição em Pauta, São Paulo, Ano XI, nº. 62, Set/Out, p. 36-42, 2003.

YAMAMOTO, D. C.; MARLET, E. F.; SILVA, F. R.; SANTOS, L. C. C. A. Caracterização das Condições Higiênico Sanitárias dos Restaurantes "fast food" de dois "Shoppings Centers", em diferentes regiões do Município de São Paulo. Rev. Higiene Alimentar, São Paulo, v. 18 - nr. 122, p. 14-19, Jul., 2004.

http://tudogostoso.uol.com.br/receita $\overline{04 / 02 / 2006}$.

. Entrevista .... 2004. Disponível em www.abrasel.com.br. Acesso em

. Uma questão para empresários e consumidores. 2006. Disponível em www.abrasel.com.br. Acesso em 05/04/2006. (Paulo Solmucci Júnior)

AMERICAN HEART ASSOCIATION. AHA Scientific Statement. Dietary guidelines. Revision 2000. A statement for health care professionals from the nutrition committee of the American Heart Assocition. Circulation 2000, no 102 , p. 2284-99, 2000.

ARAÚJO, C.M.W; BOTELHO, R.;GINANI, C.V; ARAÚJO, C.M.H; ZANDONADI, P.R. Da Alimentação a gastronomia. $1^{\mathrm{a}}$. Edição. Ed. Universidade de Brasília. Brasília, 2005.

BRANDÃO, A. P.; BRANDÃO, A. A.; MAGALHÃES, M. E. C.; POZZAN, R. Epidemiologia da Hipertensão Arterial. Rev. Soc. Cardiologia Estado de São Paulo. Vol. 13 - nr. 1, Jan/Fev., 2003. 
DUTRA, M. R. C. A Boa Mesa Mineira; um estudo de cozinha e identidade, Dissertação de mestrado apresentada ao Programa de Pós-Graduação do Museu Nacional da UFRJ, 1991.

FAUSTO, M.A. Planejamento de dietas e da alimentação. Rio de Janeiro - RJ: Editora Revinter, 2003.

FUCHS FD, MOREIRA LB, MORAES RS, BREDEMEIER M; CARDOZO SC. Prevalence of systemic arterial hypertension and associated risk factors in the Porto Alegre metropolitan area. Populational-based study. Arquivos Brasileiros de Cardiologia 63(6):473-479.

GARCIA, R. W. D. Práticas e comportamento alimentar no meio urbano: um estudo no centro da cidade de São Paulo. Cad. Saúde Pública, Rio de Janeiro, 13 (3), p. 455-467, jul-set, 1997.

GARCIA, R. W. D. Reflexos da Globalização na Cultura Alimentar: considerações sobre as mudanças na alimentação urbana. Rev. de Nutrição, Campinas, 16 (4), p. 483-494, out-dez, 2003.

GUIMARÃES, A. et al. Matéria de capa. Tendência do Food Service: Oferecer Alimentação Saudável. Rev. Nutrição em Pauta, São Paulo, Ano IX, nº 74, Mar/Abr, p. 8-14, 2001.

http://departamentos.cardiol.br/dha/ivdiretriz/slides/slides.asp

http://tudogostoso.uol.com.br/receita

Institute of Medicine (2004). Dietary Reference Intakes for water, potassium, sodium, chloride, and sulfate. Washington, DC: The National Academies Press, in press. (www.iom.edu/report.asp?id=18495). Rev. Gatorade Sports Science Institute. vol 41 Set-Out-Nov 2004. Requerimentos Nutricionais de água e sódio para adultos ativos..

JOMORI, M. M., et al. Proposta de um Método para a avaliação da Escolha Alimentar do Comensal de um Restaurante por Peso. Rev. Nutrição em Pauta, São Paulo, Ano XIII, nº 74, Set/Out, p. 45-48, 2005.

LOLIO, C. A. de. EPIDEMIOLOGÍA DA HIPERTENSÃO ARTERIAL. Rev. Saúde Pública. São Paulo 24 (5): 425-32, 1990.

MONDINI L.; MONTEIRO, C. A. Mudanças no padrão de alimentação da população urbana brasileira (1962-1988). Rev. Saúde Pública, 28 ("): 433-9, 1994. São Paulo. 
MONTEIRO, C. A; MOURA, E. C; JAIME, A. L; FLORINDO, A. A; FIGUEIREDO, I. C. R; BERNAL, R; SILVA, N. N. Monitoramento de Fatores de Risco para doenças crônicas por entrevistas telefônicas. Rev. de Saúde Pública 2005; 39(1): 47-57.

ORGANIZAÇÃO MUNDIAL DE SAÚDE. Diet, nutrition and prevention of chronic diseases. Report of a joint WHO/FAO expert consultation. Technical Report Series 916. Geneva, 2003.

ORTIZ, R. Mundialização e Cultura, São Paulo: Brasiliense, 1994.

PROENÇA, R. P. da C. Desafios Contemporâneos com Relação à Alimentação Humana. Rev. Nutrição em Pauta, São Paulo, Ano X, nº. 52, Jan/Fev, p. 32-36, 2002.

PROENÇA, R. P. da C. Qualidade Nutricional e Sensorial na Produção de Refeições. Rev. Nutrição em Pauta, São Paulo, Ano XIII, nº. 75, Nov/Dez, p. 4-14, 2005.

SENNA, T.; Araújo, W. M. C. Avaliação do hábito de lavar as mãos entre consumidores de restaurantes do tipo self service. Trabalho apresentado no I Congresso Brasileiro de Gastronomia. CET - UnB, 2004).

SESI - Departamento Regional de São Paulo. Alimente-se bem com R\$1,00. 6a Edição. Julho 2003. São Paulo.

SLOAN, D. [tradução Sonia Bidutte] Gastronomia, restaurantes e comportamento do consumidor. Barueri, SP: Manole, 2005.

TEICHMANN, I.M. Cardápios - técnicas e criatividade. 5. ed. Caxias do Sul: EDUCS, 2000. Coleção Hotelaria.

TOSCANO, C.M. As campanhas nacionais para detecção das doenças crônicas não-transmissíveis: diabetes e hipertensão arterial. Rev. Ciência e Saúde Coletiva, 9(4): 885-895, 2004.

VEIROS, M. da B., PROENÇA, R. P. da C. Avaliação Qualitativa das Preparações do Cardápio em uma Unidade de Alimentação e Nutrição - Método AQPC. Rev. Nutrição em Pauta, São Paulo, Ano XI, nº. 62, Set/Out, p. 36-42, 2003.

YAMAMOTO, D. C.; MARLET, E. F.; SILVA, F. R.; SANTOS, L. C. C. A. Caracterização das Condições Higiênico Sanitárias dos Restaurantes "fast food" de dois "Shoppings Centers", em diferentes regiões do Município de São Paulo. Rev. Higiene Alimentar, São Paulo, v. 18 - nr. 122, p. 14-19, Jul., 2004. 